\title{
Gut schlafen und entspannen
}

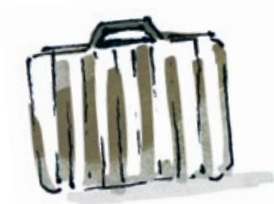

Interventionskoffer, die Neunte Wie oft liegen bei den Durchgängen im Nachtdienst immer dieselben Patienten wach und äußern:

„Ich kann nicht einschlafen“? Manche kommen auch mitten in der Nacht verzweifelt aus dem Zimmer und bitten um Hilfe, weil sie seit Stunden grübelnd wach liegen. In unserem Interventionskoffer geben wir Ihnen das nötige Handwerkszeug, damit Ihre Patienten entspannter einschlafen und ausgeruht aufwachen.

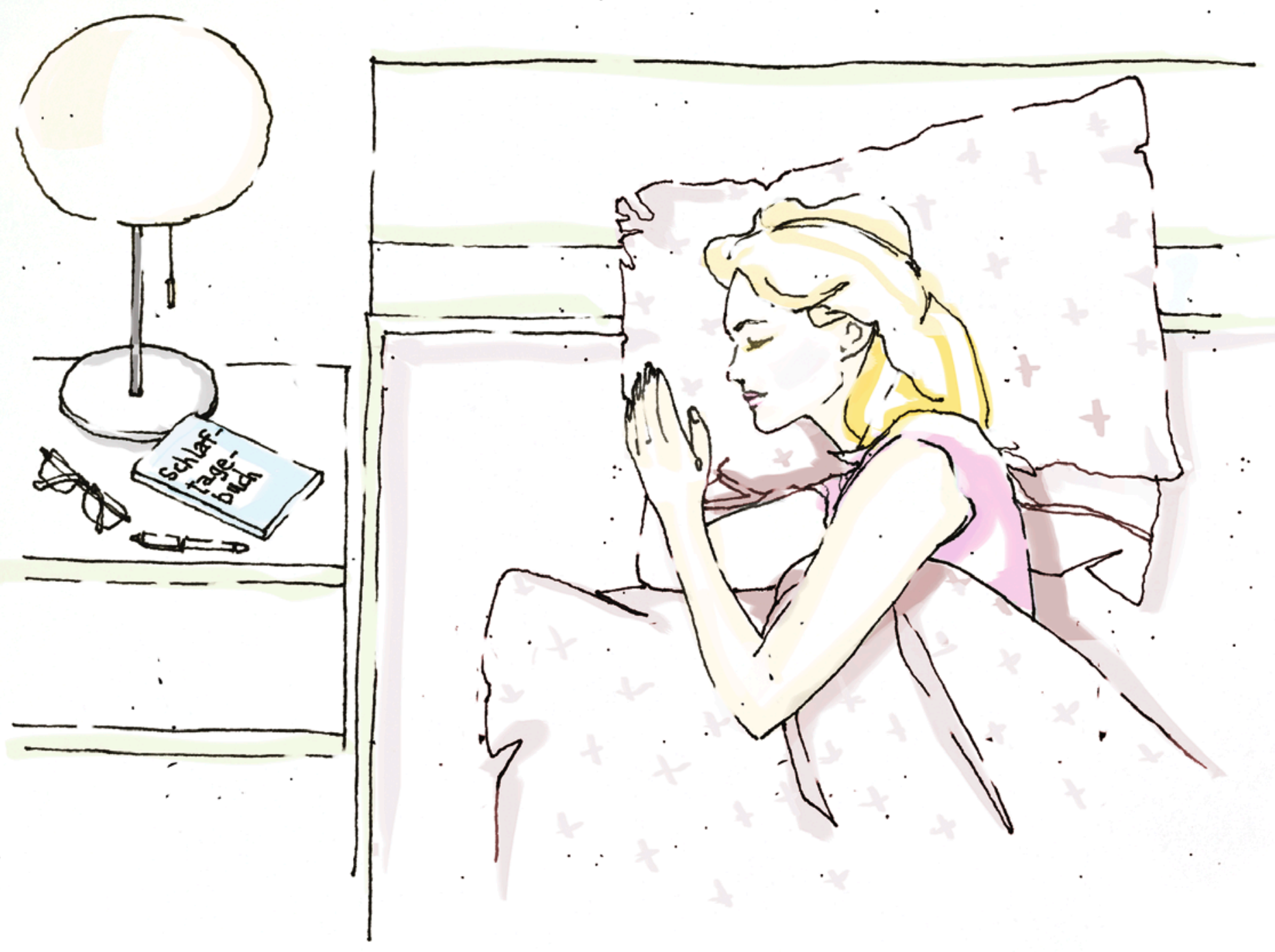


- Jessica Simianer -

Schlafstörungen sind ein Thema, mit dem die Berufsgruppe der Pflege direkt konfrontiert ist. Nicht nur im Nachtdienst, sondern auch im Stationsalltag. Bereits in der Anamnese geben die Patienten häufig an, unter Einschlaf- oder Durchschlafstörungen zu leiden. Es kann auch von Dyssomnien gesprochen werden. Studien zeigen mittlerweile sogar, dass beinahe alle Depressionen mit Schlafstörungen einhergehen. ${ }^{1}$

Diese können sich im Auftreten von Einschlafstörungen oder wiederholten intermittierenden Wachphasen zeigen. Charakteristisch ist jedoch das vorzeitige morgendliche Früherwachen, sehr häufig verbunden mit einem ausgeprägten Stimmungstief. Dabei gilt eine Veränderung der Schlafphasen ( $\rightarrow$ Info Schlafphasen) als ursächlich. ${ }^{2}$

Dies erscheint logisch, denn viele Patienten mit einer Depression leiden unter der Neigung, zu grübeln und Gedankenkreisen. Diese Symptome halten sie vom Schlafen ab und können zu Anspannung führen.

Patienten, die unter Schlafstörungen leiden, haben häufig eine Tagesmüdigkeit und den Drang, tagsüber zu schlafen. Sie leiden unter Konzentrationsstörungen und die Stimmung ist irgendwann gereizt. Dies wiederum wirkt sich auf das Verhalten der Patienten in den Therapien und Gruppenangeboten auf Station aus. Auch die Alltagsgestaltung der Patienten ist von den Folgen der Schlafstörungen betroffen. Da sich das Aufgabengebiet der psychiatrischen Pflege genau in diesem Bereich wiederfindet, liegt der Gedanke an eine pflegetherapeutische Gruppe zum Thema Schlafstörungen nahe.

Denn viele Interventionsmöglichkeiten sind in der Regel nicht bekannt. Oft wird dem Patienten ein Bedarfsmedikament angeboten und eventuell Ohropax. Vielleicht werden noch ein paar aufbauende und verständnisvolle Worte für ihn gefunden, aber damit wird er wieder in sein Bett geschickt.

Im Folgenden wird deshalb das Konzept der pflegetherapeutischen Gruppe „Gut schlafen und entspannen“ vorgestellt. Sie hat sich aus den oben erläuterten Problemen in der Praxis entwickelt. Die Gruppe soll den Patienten sowohl Interventionsmöglichkeiten bei Schlafstörungen aufzeigen, als auch eine Entspannungsmethode vermitteln, die sie bei Schlafstörungen anwenden können ( $\rightarrow$ Fallbeispiel).

\section{Gruppenmerkmale}

Unter Berücksichtigung der Raumgröße und des Zeitfensters, beschränkt sich die Größe der Gruppe auf idealerweise sechs Mitglieder. Für die Durchführung sollte eine Stunde Zeit eingeplant werden. Die Gruppe kann offen geführt werden. Dies bedeutet, dass Patienten jederzeit während des Gruppenzyklus einsteigen können.

Jedoch sollte immer ein Vorgespräch zur Einführung in die Gruppe stattfinden. Dies kann mit einer persönlichen Einladung zu der Gruppe erfolgen. Dabei kann dem Patienten ein Informationsblatt mit allen wichtigen Details und Terminen ausgehändigt werden. So werden das Interesse und die Neugier der Patienten geweckt.

\section{Gruppenziele}

Pflegetherapeutische Gruppen im Allgemeinen dienen der Förderung der Beziehungsgestaltung zu den Patienten auf der Station. Dabei können die Wirkfaktoren der Milieutherapie nach Edgar Heim als Grundlage einer allgemeinen Zielformulierung dienen.

Die Partizipation, also die Beteiligung der Patienten am Krankheitsprozess, und die offene Kommunikation werden durch das Erleben in der Gruppe gefördert. Ebenso sind das Soziale Lernen, zum Beispiel durch die Blitzlichtrunde am Ende einer Gruppe, und das Leben in der Gemeinschaft als übergeordnete Ziele einer Gruppe auf Station anzusehen. ${ }^{3}$

Bei der speziellen Zielsetzung sollte berücksichtigt werden, dass die Ziele für die Patienten realistisch sind, jedoch keine Über- oder Unterforderung darstellen. Zudem sollten die individuellen Zielvereinbarungen der Patienten im Rahmen des Pflegeprozesses berücksichtigt werden. Insbesondere eignet sich hierfür die Pflegediagnose der Schlafstörung.

Um den aufklärenden, den sogenannten edukativen Charakter der Gruppe hervorzuheben, ist es notwendig, die Sensibi-
Schlafphasen

Der Schlaf verläuft in der Regel zyklisch in fünf Phasen. Die Phasen eins bis vier werden als Non-REM-Phasen bezeichnet.

Die erste Phase wird auch Einschlafphase genannt. Dies ist ein Dämmerzustand zwischen Wachsein und leichtem Schlaf. Es besteht eine leichte Erweckbarkeit. Die zweite Phase ist der leichte Schlaf. Das Bewusstsein ist ausgeschaltet, die Augen bewegen sich nicht mehr. Der Muskeltonus im Körper ist herabgesetzt und der Körper kann sich entspannen. Diese Phase nimmt mehr als die Hälfte der gesamten Schlafzeit ein.

Die dritte Phase ist der beginnende Tiefschlaf und wird auch Delta-Schlaf genannt. Etwa eine Stunde nach dem Einschlafen wird der Schlaf tiefer. Die Augen stehen dann still und es gilt eine schwere Erweckbarkeit.

Die vierte Phase besteht aus dem Tiefschlaf.

Die fünfte Schlafphase wird als REMSchlaf bezeichnet. Sie ähnelt dem Leichtschlaf, ist aber durch schnelle Augenbewegungen unter geschlossenen Lidern gekennzeichnet. Die Erweckbarkeit ist schwer. Träume in dieser Phase sind lebhaft, fantasie- und gefühlvoll.

Die Zyklen werden bei Menschen ohne Schlafstörungen drei bis fünf Mal pro Nacht durchlaufen. Dabei ist die REMPhase in jedem Zyklus länger. Sie kann von fünf bis 50 Minuten dauern. Der Anteil des Tiefschlafs nimmt von Zyklus zu Zyklus ab. Die Dauer und Abfolge der Phasen prägen den Erholungswert des Schlafs. ${ }^{4}$

Bei depressiven Patienten beginnt der meist traumreiche und durch schnelle Augenbewegungen gekennzeichnete REM-Schlaf (Rapid-Eye-Movement) schneller, die Zeit vom Einschlafen bis zum Auftreten der ersten REM-Phase ist verkürzt, die Dauer der ersten REM-Phase jedoch deutlich verlängert. In den folgenden REM-Phasen treten häufiger schnelle Augenbewegungen auf als bei gesunden Personen. ${ }^{2}$

lisierung für das eigene Schlafverhalten als Gruppenziel festzulegen. Damit soll ein Bewusstsein für die individuelle Schlafqualität geschaffen werden. Außerdem kann 
dieses Bewusstsein präventiv als Frühwarnzeichen für die Exacerbation, also das erneute Auftreten der Symptome der psychischen Erkrankung, genutzt werden.

Ein weiteres Ziel der Gruppe ist es, ein allgemeines Wohlbefinden zu erreichen und Entspannungsgefühle im eigenen Körper positiv wahrzunehmen. Dieses Ziel bezieht sich hauptsächlich auf die Entspannungsmethode der Progressiven Muskelrelaxation (PMR) nach Jacobson. Sie dient der Achtsamkeit, einer besonderen Form der Aufmerksamkeit und Wahrnehmung, und ist bei Einschlafstörungen hilfreich.

Darüber hinaus soll die Gruppe Nervosität, Unruhe und Stress reduzieren. Dies kann im Verlauf durch eine subjektive Verbesserung der Schlafqualität und das regelmäßige Durchführen der PMR erreicht werden.

Durch die Gespräche untereinander während der einzelnen Module und der Blitzlichtrunde am Ende soll der Informationsaustausch unter den Betroffenen gefördert werden. Der persönliche Austausch benötigt Vertrauen als Grundlage und schafft dadurch ein Gefühl der Zusammengehörigkeit. Der Austausch unterstützt zudem das Bewusstsein für die Thematik und regt zu einer intensiven Auseinandersetzung an.

\section{Gruppenmodule}

Die Gruppe besteht aus insgesamt vier verschiedenen Modulen, die aufeinander folgen. Das vierte Modul, die Entspannungsmethode der PMR, findet dreimal hintereinander statt. So können sich die Abläufe der Entspannungsmethode besser bei den Patienten festigen. Dadurch fällt die erfolgreiche Anwendung der PMR leichter. Im Folgenden werden der Ablauf und Inhalt der einzelnen Module grob skizziert.

\section{Modul Schlaftagebuch}

Um ein Bewusstsein bei den Patienten für das eigene Schlafverhalten zu schaffen, ist das Führen eines Schlaftagebuchs hilfreich $(\rightarrow$ Abb. 1). Dieses wurde eigenhändig für die Gruppe „Gut schlafen und entspannen“ entwickelt.

Den Gruppenmitgliedern wird das Tagebuch ausgehändigt, mit der Empfehlung, es auf dem Nachttisch zu platzieren und vor dem Schlafengehen und nach dem Auf- stehen zu führen. Dabei sollen die spontanen Antworten notiert werden, sodass die Bearbeitung der abgefragten Items keinen Druck erzeugt. Die Bedeutung der einzelnen Punkte des Tagebuchs wird gegebenenfalls erklärt.

Zur nächsten Gruppe soll das Tagebuch mitgebracht und die Ergebnisse besprochen werden. Die Teilnehmer der Gruppe erhalten jede Woche ein neues Schlaftagebuch. Zu Beginn jeder Gruppensitzung wird kurz auf das individuelle Schlafverhalten der vergangenen sieben Nächte eingegangen. Dies lernen die Patienten mit Hilfe des Tagesbuchs spezifisch zu schildern.

\section{Modul Schlafphasen}

Dieses Modul dient dazu, zu verstehen, wozu der Körper Schlaf benötigt und welche negativen Folgen Schlafstörungen haben können. Durch die theoretische Wissensvermittlung der Schlaf- und damit verbundenen Erholungsphasen des Körpers wird ein Bewusstsein für das eigene Schlafverhalten geschaffen $(\rightarrow$ Info Schlafphasen).

\section{Modul Schlafhygiene}

Mit den Regeln der Schlafhygiene sollen Möglichkeiten vorgestellt werden, die das Ein- und Durchschlafen fördern. Hierbei können auch die Schlaftagebücher miteinbezogen werden. Die Schlafhygieneregeln stellen eine Alternative oder Ergänzung zu der medikamentösen Behandlung von Schlafstörungen dar, beispielsweise

- feste Aufsteh- und Zubettgehzeiten;

- das Durchführen fester Rituale vor dem Schlafengehen;

- das Vermeiden von größeren Nahrungs- und Trinkmengen vor dem Zubettgehen;

- Verzicht auf Genussmittel, wie Alkohol, vor dem Schlafengehen;

- das Schlafzimmer und Bett nur zum Schlafen nutzen und nicht etwa als Arbeitszimmer;

- die Gedanken und Aktivitäten des Tages etwa eine Stunde vor dem Schlafengehen ablegen; in diesem Zusammenhang kann auch auf die Technik des Gedankenstopps aus der kognitiven Verhaltenstherapie verwiesen werden;
Fallbeispiel

Frau $X$ leidet unter einer schweren depressiven Episode mit einhergehenden Ein- und Durchschlafstörungen. Diese lassen mit einer medikamentösen Behandlung der Erkrankung zunächst nach. Als die Medikamente reduziert werden, treten bei Frau X erneut Schlafstörungen auf. Zudem ist sie von einer inneren Unruhe beeinträchtigt.

Ein therapeutischer Schwerpunkt ist, Frau $X$ für das eigene Schlafverhalten zu sensibilisieren. Außerdem kann sie Alternativen zur medikamentösen Behandlung von Schlafstörungen kennenlernen und bei der Anwendung unterstützt werden. Dazu kommt, dass Frau X in der „Gut schlafen und entspannen“-Gruppe ein Muskelrelaxationsverfahren kennenlernen kann. Dies ist bei regelmäßiger Übung auch bei innerer Unruhe anwendbar.

- beim nächtlichen Erwachen nicht auf den Wecker schauen, um Stress zu vermeiden. ${ }^{4}$

Im Hinblick auf das nächste Modul, kann bereits hier erwähnt werden, dass das Erlernen und Anwenden einer Entspannungsmethode hilfreich gegen Ein- und Durchschlafstörungen sein kann.

\section{Modul Progressive Muskelrelaxation nach Jacobson}

Diese Entspannungsmethode findet im Liegen auf Matten oder im Sitzen auf Relaxsesseln statt. Die Patienten werden zu entspannter Hintergrundmusik angeleitet, einzelne Muskelgruppen anzuspannen und zu entspannen. Dabei liegt der Schwerpunkt auf dem Gefühl der Entspannung. Um die Entspannungsmethode selbstständig anwenden zu können, ist eine regelmäßige Übung notwendig.

Nach dreimaliger Wiederholung dieses Moduls beginnt der Zyklus von neuem mit dem Modul des Schlaftagebuchs.

\section{Evaluation}

Die Evaluation der Gruppenziele, insbesondere des allgemeinen Wohlbefindens, kann durch die Reflexion der Patienten über ihr eigenes Schlafverhalten erfolgen. Darüber hinaus sind die Schlaftagebücher ein wertvolles Assessment zur 


\begin{tabular}{|c|c|c|c|c|c|c|c|}
\hline \multicolumn{8}{|l|}{ Abends } \\
\hline Tag & Montag & Dienstag & Mittwoch & Donnerstag & Freitag & Samstag & Sonntag \\
\hline \multicolumn{8}{|l|}{ Datum } \\
\hline \multicolumn{8}{|l|}{ Zubettgehzeit } \\
\hline \multicolumn{8}{|l|}{ Schlaf tagsüber } \\
\hline \multicolumn{8}{|l|}{ Alkoholkonsum } \\
\hline \multicolumn{8}{|l|}{$\begin{array}{l}\text { Letztes koffeinhaltiges } \\
\text { Getränk }\end{array}$} \\
\hline \multicolumn{8}{|l|}{$\begin{array}{l}\text { Eingenommene } \\
\text { Medikation zur Nacht }\end{array}$} \\
\hline \multicolumn{8}{|l|}{ Besondere Belastungen } \\
\hline \multicolumn{8}{|l|}{$\begin{array}{l}\text { Aktivitäten der letzten } \\
30 \text { Minuten }\end{array}$} \\
\hline Sonstiges & & & & & & & \\
\hline
\end{tabular}

ten mit dem Thema fühlen sich die Patienten wertgeschätzt und angehört. Das Thema Schlafstörungen ist weit verbreitet, jedoch ist dies nur wenigen Patienten bewusst. Die Erleichterung darüber ist jedes Mal deutlich spürbar, wenn die Patienten feststellen, dass sie mit ihren Problemen nicht alleine sind und es Hoffnung auf Besserung gibt.

\begin{tabular}{|l|l|l|l|l|l|l|l|}
\hline Morgens \\
\hline Tag & Montag & Dienstag & Mittwoch & Donnerstag & Freitag & Samstag & Sonntag \\
\hline Datum & & & & & & & \\
\hline Uhrzeit beim Aufwachen & & & & & & & \\
\hline Uhrzeit beim Aufstehen & & & & & & & \\
\hline Geschätzte Einschlafdauer & & & & & & & \\
\hline $\begin{array}{l}\text { Aufwachhäufigkeit in } \\
\text { letzter Nacht }\end{array}$ & & & & & & & \\
\hline $\begin{array}{l}\text { Wachzeiten letzter } \\
\text { Nacht }\end{array}$ & & & & & & & \\
\hline $\begin{array}{l}\text { Aktuelles Befinden } \\
1=\odot 2=\Theta 3=\odot\end{array}$ & & & & & & & \\
\hline Sonstiges & & & & & & & \\
\hline
\end{tabular}

\section{Literatur}

1 Berger M. Psychische Erkrankungen. München: Urban \& Fischer Verlag; 2015

2 Möller HJ, Laux G, Kapfhammer HP. Psychiatrie, Psychosomatik, Psychotherapie: Band 1 Allgemeine Psychiatrie, Band 2 Spezielle Psychiatrie. Heidelberg: Springer Verlag; 2011

3 Rakel T, Lanzenberger A. Pflegetherapeutische Gruppen in der Psychiatrie. Stuttgart: Wissenschaftliche Verlagsgesellschaft; 2009

4 Sauter D et al. Lehrbuch Psychiatrische Pflege. Bern: Verlag Hans Huber; 2015

Abb. 1 Schlaftagebuch (eigene Darstellung).

Überprüfung des Schlafverhaltens und der Schlafqualität. Diese Ergebnisse fließen wiederum in die Evaluation der Pflegediagnosen mit ein. Des Weiteren kann durch die Gruppenleitung die Entwicklung der sozioemotionalen Kompetenzen beobachtet und evaluiert werden.

\section{Erfahrungsaustausch}

Es ist wichtig, den Patienten die Möglichkeit für einen Erfahrungsaustausch zu diesem Thema zu schaffen. Sie haben in der Gruppe die Gelegenheit, ihren bisherigen Umgang mit Schlafstörungen zu reflektieren. Der Austausch untereinander führt zu neuen Denkanstößen und Ideen, um die persönliche Schlafhygiene weiterzuentwickeln. Die Gruppenmitglieder profitieren von den Erfahrungen der Anderen und werden dadurch motiviert, ihr bisheriges Schlafverhalten zu verändern.

Außerdem stellt die Gruppe eine wertvolle Gelegenheit zur Psychoedukation und zur Beziehungsgestaltung zu den Mitgliedern dar. Durch das Berichten von den bisherigen Erfahrungen und Schwierigkei-

\section{Autorin}

\section{Jessica Simianer}

Gesundheits- und Krankenpflegerin in der Tagesklinik für Menschen der zweiten Lebenshälfte des Klinikums Ludwigsburg; Fachweiterbildung für Psychiatrie E-Mail: jessicasimianer@gmail.com

\section{Bibliografie}

DOI 10.1055/s-0043-108816

PPH 2017; 23: 162-165

(c) Georg Thieme Verlag KC

Stuttgart · New York · ISSN 0949-1619

Seit dem Bestehen der Gruppe ist der Umgang mit dem Thema Schlafstörungen auf Station deutlich offener und transparenter geworden. Die Patienten sprechen auch außerhalb der Gruppe darüber und tauschen sich mit anderen Patienten und Mitgliedern des multiprofessionellen Teams über das Thema aus.

Die Durchführung der Gruppe gibt auch der Pflegekraft die Gelegenheit, sich mit dem eigenen Schlafverhalten auseinanderzusetzen. Dies kann durch die Rahmenbedingungen im Pflegealltag durchaus störanfällig sein. Daher ist es gut, dass die Inhalte der einzelnen Module auch eins zu eins selbst angewendet werden können. Somit dient die Gruppe gleichzeitig der persönlichen Gesundheitsprävention.

Durch das Bewusstsein, dass jeder von Schlafstörungen betroffen sein kann, fällt schließlich ein empathisches, wertschätzendes und echtes Auftreten in der Gruppe nicht mehr schwer. 